\title{
Single-bounce Ellipsoidal Capillary for X-ray microscopes: Design and Measurements
}

\author{
Yudan Wang ${ }^{1}$, Yuqi Ren ${ }^{1}$, Tianxi $\operatorname{Sun}^{2}$, Fen Tao $^{1}$, Biao Deng $^{1, *}$, and Tiqiao Xiao ${ }^{1, *}$ \\ 1. Shanghai Institute of Applied Physics, Chinese Academy of Sciences, Shanghai 201204, China \\ 2. The Key Laboratory of Beam Technology and Materials Modification of the Ministry of Education, \\ Beijing Normal University, Beijing 100875, China \\ * Corresponding author: dengbiao@sinap.ac.cn
}

Full field transmission X-ray microscopes (TXM) are conveniently adapted for three-dimensional computed tomography due to the large depth of focus of the X-ray lenses, which is one of the most powerful tools for observation of the inner structure of objects at nanoscale. The condenser is the key component of the TXM system. The condenser focuses the X-ray beam to the sample and increase the flux density, while keeping the numerical-aperture match with the zone plates. There are three kind of condenser optics for TXM. Beam shaper is consists of square subfields of a linear grating with constant line orientation and period, which has a low efficiency [1]. X-ray compound refractive lenses could be fabricated with acceptable efficiency [2]. However, it is sensitive with energy. Single-bounce hollow capillaries with ellipsoidal shapes are efficient, rugged, easy to align, and achromatic, clearly superior to other condensers, which is suitable for TXM system [3-4]. Due to the high efficiency, the single-bounce ellipsoidal capillary is the best choice of condenser for the TXM at this beamline. Up to now, many tabletop and synchrotron X-ray microscopes uses the ellipsoidal capillary as the condenser. The Three Dimensional Nano-imaging Beamline at Shanghai Synchrotron Radiation Facility (SSRF) phase II project will be built with TXM of a spatial resolution of $20 \mathrm{~nm}$. SSRF is developing the capillary with Beijing Normal University.

The X-rays are reflected off the internal surface of the capillary, which is an appropriate ellipsoidal shape. The capillaries are limited in NA only by the critical angle for grazing incidence. A single-bounce ellipsoidal capillary was designed for the TXM system at the BL13W1 beamline at SSRF. The design goal of the microscope is a spatial resolution of $100 \mathrm{~nm}$ at $9 \mathrm{keV}$. The zone plate with the diameter of $100 \mu \mathrm{m}$ and the outmost width of $70 \mathrm{~nm}$ was chosen to be objective lens. It has the NA of $0.98 \mathrm{mrad}$. The sample is installed $34 \mathrm{~m}$ downstream from the source. Table 1 shows the designed parameters of the capillary. The capillary, named with BNU 5\#, was fabricated by Beijing Normal University.

The quality of capillaries was estimated first by optical measurement. The outer line of capillary was measured with a laser beam diameter gauge (including the Optical micrometer of LS-70100M) with the scanning interval of $0.2 \mathrm{~mm}$. Then the inner line was calculated using the inner/outer diameter ratio. The entrance and exit radius, length and inner surface of capillary BNU 5\# can be obtained, as shown in Figure 1 (a). It is calculated that the capillary has a slope error of $30 \mu \mathrm{rad} \mathrm{rms}$.

An X-ray online test at BL13W1 Beamline is carried out to evaluate the performances of the capillaries, including reflected rings, focal size, focal length, depth of focal, efficiency, divergence angle of outer ring. For focal length measurement, the detector is positioned after the capillary and scanned near the focal point to find the smallest spot size. The depth of focal is the length of acceptable focus which is two times of focal size. The reflected beam is also obtained at different places downstream of the capillary to observe the far-field intensity distribution, as shown in Figure 2 (a-b). The reflected ring is not very uniform. Table 1 is the measured parameters of capillary BNU 5\#. The divergence angle implies the performance of the 
capillary in the X-ray microscope in the "as designed" configuration. It is measured to be $1.75 \mathrm{mrad}$, shown in Figure $1(\mathrm{~b})$, matches the NA of zone plate $(1.75 \mathrm{mrad}=0.95 * 2 * \mathrm{NA})$, which is good agreement with the design configuration. The reflection efficiency is another important parameter to characterize the performance of the capillary. It is the ratio of all the reflected X-ray photons to the total incident photons intercepted by the ellipsoid. The reflection efficiency is affected by the surface smoothness, the X-ray grazing angle, and the critical angle of the surface materials. Reflection efficiency of BNU 5\# was measured at $9 \mathrm{keV}$, which is about $83 \%$.

The capillary BNU 5\# was used as condenser in X-ray microscopy. As the emittance of BL13W1 is too small to match the microscope acceptance, hence either only part of the sample area is illuminated. An $\mathrm{X} / \mathrm{Z}$ wobbling system is used to scan the capillary to overcome the problem. Fig. 2 (c-d) shows the X-ray transmission image of a test pattern in a microscope at BL13W1 Beamline with a resolution of better than $100 \mathrm{~nm}$. The FOV is larger and much more uniform with the cost of longer exposure time while wobbling.

Preliminary results demonstrated that high-quality single-bounce capillary condensers is developed for Xray microscopes. The capillary has been used successfully for synchrotron X-ray microscopes and the spatial resolution of $100 \mathrm{~nm}$ is obtained. It can be helpful for developing capillary condensers for the nanoimaging beamline at SSRF phase II project.

\section{References:}

$1 \quad$ K. Jefimovs et al, J Synchrotron Radiat 15, 106 (2008).

$2 \quad$ H Stollberg et al, Rev Sci Instrum 77 (12), 33 (2006).

$3 \quad$ X. H. Zeng et al, Appl Optics 47 (13), 2376 (2008).

$4 \quad$ R. Huang et al, J Synchrotron Radiat 13, 74 (2006).

Table 1 Designed and measured parameters of ellipsoidal capillary

\begin{tabular}{|c|c|c|c|c|c|}
\hline \multicolumn{3}{|c|}{ Designed parameters } & \multicolumn{2}{c|}{ Measured parameters } \\
\hline Semi major axis a $(\mathrm{mm})$ & 17000 & Semi minor axis $\mathrm{b}(\mathrm{mm})$ & 1.148 & Spot size $/ \mu \mathrm{m}$ & 40 \\
\hline Entry radius $\mathrm{H}(\mu \mathrm{m})$ & 202.4 & Exit radius $\mathrm{h}(\mu \mathrm{m})$ & 157.5 & Focal length/mm & 155 \\
\hline Length $\mathrm{L}(\mathrm{mm})$ & 105.7 & Working distance $(\mathrm{mm})$ & 160.67 & Focal depth/mm & 10 \\
\hline
\end{tabular}
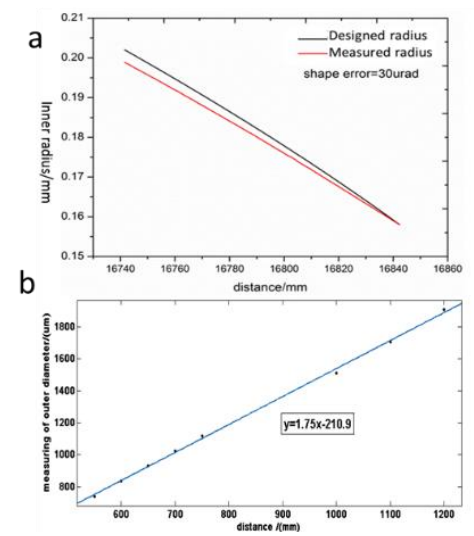

Figure 1 (a) the design radius and measured data. (b) the divergence angle of the outer rings.

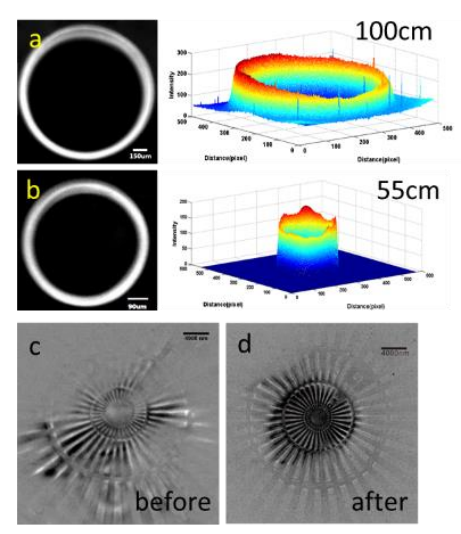

Figure $2(a-b)$ Condenser reflected pattern and the intensity profile. (c-d) TXM images before and after wobbling. 